\title{
Muscle depletion and the prediction of chemotherapy toxicity
}

\author{
Maurizio Muscaritoli • Alessio Molfino • \\ Filippo Rossi Fanelli
}

Received: 6 May 2013/Accepted: 28 May 2013/Published online: 11 June 2013

(C) SIMI 2013

During the past few decades, while the death rate for heart disease has declined steadily, cancer death rates have remained relatively constant [1]. Moreover, cancer is becoming a disease associated with aging, with patients aged 65 years and older having an 11-fold increase in cancer incidence and a 16-fold increase in cancer mortality when compared to individuals younger than $65[2,3]$. The population of older adults is rapidly growing worldwide, and it is estimated that the number of people older than 65 will exceed $20 \%$ by 2030 in the United States. Furthermore, aging is paralleled by an increase in comorbidities, which increases patients' vulnerability [4]. This will progressively increase the need for cost-effective antineoplastic strategies for an increasing population of patients.

Part of the limited efficacy of cancer treatments is related to chemotherapy toxicity [5-7]. Up to $30 \%$ of patients treated with 5-fluorouracil (5-FU)/capecitabine experience dose-limiting toxicities including diarrhea, handfoot syndrome, mucositis and myelosuppression [8]. Toxicity frequently implies reduction or interruption of anticancer therapy and may lead to fatal complications, particularly in older persons [3, 9]. Besides its detrimental role in negatively affecting prognosis and survival of cancer patients, toxicity to chemotherapy negatively impacts on the financial burden of cancer, causing increase in both direct medical costs and indirect mortality costs [10] An American pilot study [11] evaluating the total costs

This comment refers to the article available at doi:10.1007/s11739-013-0936-8.

M. Muscaritoli $(\bowtie) \cdot$ A. Molfino · F. Rossi Fanelli

Department of Clinical Medicine, Sapienza University of Rome,

Viale dell'Università, 37, 00185 Rome, Italy

e-mail: maurizio.muscaritoli@uniroma1.it of chemotherapy-induced toxicity in ovarian cancer patients in 2001 estimates that the cost for every single episode of neutropenia is USD $\$ 7,546$. In a subsequent study from the same group [12], the cost per episode of febrile neutropenia in patients receiving inpatient care ranges between USD $\$ 12,150$ and USD \$21,601. Thus, the control of chemotherapy-induced toxicity has become a major healthcare issue, and systems or models for the prompt and effective identification of patients at risk are strongly required. The study of mutations in metabolic enzymes involved in drug metabolism represents an example of such predictive approach.

Drug-metabolizing enzymes play a central role in the metabolism, elimination and detoxification of drugs introduced into the body [13]. Therefore, genetic variability is crucial in determining the probability of the occurrence of chemotherapy toxicity in cancer patients. Dihydropyrimidine dehydrogenase (DPD) is the rate-limiting enzyme in the catabolism of fluoropyrimidines, like capecitabine and 5-FU, converting $>80 \%$ of an administered dose of 5-FU to inactive metabolites [14]. Deficiency of DPD activity is associated with severe toxicities, sometimes fatal, following administration of 5-FU class drugs. Molecular defects of the dihydropyrimidine dehydrogenase gene (DPYD) lead to the deficiency of DPD activity and cause this clinically severe syndrome [15].

In their study published in this issue of Internal and Emergency Medicine, Magnani et al. [16] analyzed 180 cancer patients as candidates for treatment with 5-FU class drugs for the most common DPD mutation, i.e. IVS14 $+1 \mathrm{G}>\mathrm{A}$ and detected four heterozygous individuals. In three out of the four patients, who had been scheduled for a $50 \%$ dose reduction to prevent side-effects, severe toxicity occurred, resulting in need for hospitalization and premature discontinuation of treatment. The authors 
therefore suggest that further dose reductions or choice of alternative treatments are warranted for patients carrying this gene mutation, underscoring the progressive need of medical oncology to rapidly shift towards a personalized medicine for cancer patients [17].

Factors others than genetic variability for key metabolic enzymes may also affect tolerance to antineoplastic treatments, particularly in older adults bearing multiple comorbidities $[3,18]$. In recent years, the role played by malnutrition, reduced lean body mass (LBM) and sarcopenia in decreasing survival, quality of life and tolerance to treatments in cancer patients has gained progressive attention [19]. Reduced LBM and sarcopenia are common features in elderly people and in patients with chronic diseases, including cancer [20]. Prado et al. [21, 22] recently reported that sarcopenia is a significant predictor of toxicity and time to tumor progression in metastatic breast cancer patients treated with capecitabine and that that low LBM is a significant predictor of toxicity in women administered 5-FU for stage II/III colon cancer. According to the authors' interpretation, reduced LBM implies a low volume of distribution of cytotoxic chemotherapy drugs and will result in higher concentrations in a shorter period of time. A reduced clearance of the drug from the systemic circulation in turn induces increased toxicity [21, 22]. In normal conditions LBM represents $80-85 \%$ of body weight, with muscle mass accounting for about $45 \%$ of body mass, thus it is reasonable that muscle mass may significantly contribute to the volume of distribution of hydrophylic drugs, such as 5-FU. An alternative to Prado's hypothesis, which is currently being tested by our group, is that sarcopenia per se determines a reduction in the total body DPD activity, in turn responsible for increased toxicity. This reasoning is based on the observation that, although the liver and lymphocytes display highest DPD activity, significant DPYD gene expression has been documented in skeletal muscle [23]. Moreover, geriatric assessment variables (ability to walk one block, decreased activities of daily living, falls in the last 6 months), which are well-known sarcopenia-related predictors of morbidity and mortality [24], significantly predict chemotherapy toxicity in older adults [18].

Approaches to cancer treatment are progressively becoming more complex and costly. Besides, cancer is nowadays a disease associated with aging, therefore making the majority of the cancer population particularly vulnerable, due to the presence of one or more chronic comorbidities. Sarcopenia frequently complicates aging and chronic conditions and causes frailty and increased vulnerability [20, 24, 25]. A careful assessment for the presence of malnutrition, sarcopenia and changes in body composition represents a cost-effective measure of personalized medicine that may help predicting chemotherapy toxicity and improving quality of life and survival rate of cancer patients.

Conflict of interest The authors have no conflict of interest.

\section{References}

1. Siegel R, Ward E, Brawley O, Jemal A (2011) Cancer statistics, 2011: the impact of eliminating socioeconomic and racial disparities on premature cancer deaths. CA Cancer J Clin 6:212-236

2. Smith BD, Smith GL, Hurria A, Hortobagyi GN, Buchholz TA (2009) Future of cancer incidence in the United States: burdens upon an aging, changing nation. J Clin Oncol 27:2758-2765

3. Monfardini S, Falci C, Brunello A, Lonardi S, Basso U (2011) Cancer in the elderly. Intern Emerg Med 6(Suppl 1):115-118

4. Piccirillo JF, Tierney RM, Costas I et al (2004) Prognostic importance of comorbidity in a hospital based cancer registry. JAMA 291:2441-2447

5. Albini A, Donatelli F, Focaccetti C, D'Elios MM, Noonan DM (2012) Renal dysfunction and increased risk of cardiotoxicity with trastuzumab therapy: a new challenge in cardio-oncology. Intern Emerg Med 7:399-401

6. Russo G, Cioffi G, Di Lenarda A et al (2012) Role of renal function on the development of cardiotoxicity associated with trastuzumab-based adjuvant chemotherapy for early breast cancer. Intern Emerg Med 7:439-446

7. Raschi E, De Ponti F (2012) Cardiovascular toxicity of anticancer-targeted therapy: emerging issues in the era of cardiooncology. Intern Emerg Med 7:113-131

8. Saif MW, Syrigos K, Mehra R, Mattison LK, Diasio RB (2007) Dihydropyrimidine dehydrogenase deficiency (DPD) in GI malignancies: experience of 4-years. Pak J Med Sci Q 23: 832-839

9. Muss HB, Berry DA, Cirrincione C, Cancer and Leukemia Group B Experience et al (2007) Toxicity of older and younger patients treated with adjuvant chemotherapy for node-positive breast cancer: the Cancer and Leukemia Group B experience. J Clin Oncol 25:3699-3704

10. American Cancer Society (2006) Cancer Facts and Figures 2006. http://www.cancer.org/research/cancerfactsfigures/cancerfacts figures/cancer-facts-figures-2006

11. Calhoun EA, Chang CH, Welshman EE, Fishman DA, Lurain JR, Bennett CL (2001) Evaluating the total costs of chemotherapyinduced toxicity: results from a pilot study with ovarian cancer patients. Oncologist 6:441-445

12. Bennett CL, Calhoun EA (2007) Evaluating the total costs of chemotherapy-induced febrile neutropenia: results from a pilot study with community oncology cancer patients. Oncologist 12:478-483

13. Meyer UA (1996) Overview of enzymes of drug metabolism. J Pharmacokinet Biopharm 24:449-459

14. Heggie GD, Sommadossi JP, Cross DS, Huster WJ, Diasio RB (1987) Clinical pharmacokinetics of 5-fluorouracil and its metabolites in plasma, urine, and bile. Cancer Res 47:2203-2206

15. Saif MW (2013) Dihydropyrimidine Dehydrogenase Gene (DPYD) Polymorphism among Caucasian and non-Caucasian Patients with 5-FU- and Capecitabine-related Toxicity Using Full Sequencing of DPYD. Cancer Genomics Proteomics 10:89-92

16. Magnani E, Farnetti E, Nicoli D, et al (2013) Fluoropyrimidine toxicity in patients with dihydropyrimidine dehydrogenase splice site variant: the need for further revision of dose and schedule. Intern Emerg Med (Epub ahead of print). PubMed PMID: 23585145 
17. Schilsky RL (2009) Personalizing cancer care: American Society of Clinical Oncology presidential address 2009. J Clin Oncol 27:3725-3730

18. Hurria A, Togawa K, Mohile SG et al (2011) Predicting chemotherapy toxicity in older adults with cancer: a prospective multicenter study. J Clin Oncol 29:3457-3465

19. Muscaritoli M, Molfino A, Gioia G, Laviano A, Rossi Fanelli F (2011) The "parallel pathway": a novel nutritional and metabolic approach to cancer patients. Intern Emerg Med 6:105-112

20. Muscaritoli M, Lucia S, Molfino A, Cederholm T, Rossi Fanelli F (2012) Muscle atrophy in aging and chronic diseases: is it sarcopenia or cachexia? Intern Emerg Med (Epub ahead of print). PubMed PMID: 22773188

21. Prado CM, Baracos VE, McCargar LJ et al (2009) Sarcopenia as a determinant of chemotherapy toxicity and time to tumor progression in metastatic breast cancer patients receiving capecitabine treatment. Clin Cancer Res 15:2920-2926
22. Prado CM, Baracos VE, McCargar LJ et al (2007) Body composition as an independent determinant of 5-fluorouracil-based chemotherapy toxicity. Clin Cancer Res 13:3264-3268

23. Nishimura M, Naito S (2006) Tissue-specific mRNA expression profiles of human phase I metabolizing enzymes except for cytochrome $\mathrm{P} 450$ and phase II metabolizing enzymes. Drug Metab Pharmacokinet 21:357-374

24. Reuben DB, Rubenstein LV, Hirsch SH et al (1992) Value of functional status as a predictor of mortality: results of a prospective study. Am J Med 93:663-669

25. Fielding RA, Vellas B, Evans WJ et al (2011) Sarcopenia: an undiagnosed condition in older adults. Current consensus definition: prevalence, etiology, and consequences. International working group on sarcopenia. J Am Med Dir Assoc 12:249-256 